Bull. Austral. Math. Soc.

$90 \mathrm{c} 30$

VoL. 56 (1997) [25-36]

\title{
MULTIOBJECTIVE SYMMETRIC DUALITY WITH INVEXITY
}

\author{
T.R. Gulati, I. Husain AND A. Ahmed
}

\begin{abstract}
Usual duality results are proved for Wolfe and Mond-Weir type multiobjective symmetric dual problems without nonnegativity constraints under invexity/generalised invexity assumptions. Moreover, assuming the kernel function to be skew symmetric, the multiobjective problems are exhibited to be self duals.
\end{abstract}

\section{INTRODUCTION}

Symmetric duality in mathematical programming in which the dual of the dual is the primal was first introduced by Dorn [5]. Subsequently, Dantzig, Eisenberg and Cottle [4], Chandra and Husain [2], Mond and Weir [10] and others cited in these references developed significantly the notion of symmetric duality. The Wolfe dual models, presented in [4], involve a scalar kernel function $K(x, y), x \in R^{n}, y \in R^{m}$, which is required to be convex in $x$ for fixed $y$ and concave in $y$ for fixed $x$ while in [10] for Mond-Weir dual models, $K(x, y)$ is assumed to be pseudoconvex/pseudoconcave. Mond and Cottle [8] showed that under the skew symmetry of $K(x, y)$, the pair of symmetric dual problems of [4] becomes self dual. Mond and Weir [10] also deduced a similar result for their models.

Hanson [7] introduced the concept of invexity. Since then many duality results which previously required convexity/generalised convexity assumptions have been extended by using invexity/generalized invexity. Recently Smart and Mond [11] applied invexity to symmetric dual problems of [9] omitting nonnegativity constraints but with an additional assumption on the invexity.

In this paper, we apply invexity/generalised invexity to Wolfe and Mond-Weir type symmetric dual multiobjective problems without nonnegativity constraints, occurring in the symmetric dual models formulated by Weir and Mond [12], but invexity and generalised invexity bear an additional restriction in the spirit of [11]. Self duality for our problems is also incorporated.

Received 1st August 1996

The work of the third author was carried out at the University of Roorkee, Roorkee, India under the study leave programme.

Copyright Clearance Centre, Inc. Serial-fee code: 0004-9729/97 $\$ A 2.00+0.00$. 


\section{Prerequisites}

Let $K(x, y): R^{n} \times R^{m} \rightarrow R^{p}$ denote a twice differentiable function and $\nabla_{y} K(x, y)$ the $m \times p$ matrix of first order partial derivatives. For the scalar function $\lambda^{T} K$ with $\lambda \in R^{p}, \nabla_{x}\left(\lambda^{T} K\right)$ and $\nabla_{y}\left(\lambda^{T} K\right)$ denote gradient (column) vectors with respect to $x$ and $y$ respectively; and $\nabla_{y y}\left(\lambda^{T} K\right)$ and $\nabla_{y x}\left(\lambda^{T} K\right)$ denote respectively the $m \times m$ and $n \times m$ matrices of second order partial derivatives.

The following ordering relations in $R^{n}$ are recalled for our use. If $x, u \in R^{n}$, then

$$
\begin{aligned}
& x \geqslant u \Leftrightarrow x^{i} \geqslant u^{i}, \quad i \in\{1,2, \ldots, n\}, \\
& x \gtrsim u \Leftrightarrow x^{i} \geqslant u^{i}, \quad i \in\{1,2, \ldots, n\}, \text { and } x \neq u, \\
& x>u \Leftrightarrow x^{i}>u^{i}, \quad i \in\{1,2, \ldots, n\} .
\end{aligned}
$$

Consider the usual constrained multiobjective optimisation problem.

(VP): Minimise $f(x)=\left(f^{1}(x), f^{2}(x), \ldots, f^{p}(x)\right)$

Subject to

$$
x \in X=\left\{x \in R^{n} \mid g(x) \leqslant 0\right\} .
$$

where $f: R^{n} \rightarrow R^{p}$ and $g: R^{n} \rightarrow R^{m}$.

Definition 1: (Geoffrion [6].) A point $x_{0} \in X$ is said to be an efficient solution of (VP) if there exists no other feasible point $x \in X$ such that $f(x) \lesssim f\left(x_{0}\right)$.

A point $x_{0}$ is said to be a properly efficient solution of (VP) if it is efficient and if there exists a scalar $M>0$ such that for each $i \in\{1,2, \ldots, p\}$ and $x \in X$ satisfying $f^{i}(x)<f^{i}\left(x_{0}\right)$, we have,

$$
\frac{f^{i}\left(x_{0}\right)-f^{i}(x)}{f^{j}(x)-f^{j}\left(x_{0}\right)} \leqslant M,
$$

for some $j$ such that $f^{j}(x)>f^{j}\left(x_{0}\right)$.

An efficient point that is not properly efficient is said to be improperly efficient. Thus $x_{0}$ is improperly efficient means that for every scalar $M>0$ (no matter how large) there is a point $x \in X$ and an $i$ such that $f^{i}(x)<f^{i}\left(x_{0}\right)$ and

$$
\frac{f^{i}\left(x_{0}\right)-f^{i}(x)}{f^{j}(x)-f^{j}\left(x_{0}\right)}>M
$$

for all $j$ satisfying $f^{j}(x)>f^{j}\left(x_{0}\right)$.

Definition 2: (Borwein [1].) A point $x_{0} \in X$ is said to be weak efficient for (VP), if there exists no other point $x \in X$ with $f(x)<f\left(x_{0}\right)$.

It readily follows that if $x_{0} \in X$ is efficient, then it is also weak efficient.

Definition 3: A function $\phi: R^{n} \rightarrow R$ is said to be invex with respect to $\eta$ if there exists a vector function $\eta: R^{n} \times R^{n} \rightarrow R^{n}$ such that for each $x$ and $u$ in $R^{n}$,

$$
\phi(x)-\phi(u) \geqslant \eta^{T}(x, u) \nabla_{x} \phi(u) .
$$


The function $\phi$ is said to be pseudo-invex with respect to $\eta$ if there exists a vector function $\eta(x, u) \in R^{n}$ such that for each $x$ and $u$ in $R^{n}$,

$$
\eta^{T}(x, u) \nabla_{x} \phi(u) \geqslant 0 \Rightarrow \phi(x) \geqslant \phi(u) .
$$

\section{WOLFE TYPE SYMMETRIC DUALITY}

In this section, we present Wolfe type symmetric dual problems and establish weak and strong duality theorems.

Consider the following pair of symmetric dual problems:

Primal Problem.

(WP): Minimise $K(x, y)-\left[y^{T} \nabla_{y}\left(\lambda^{T} K\right)(x, y)\right] e$ Subject to

$$
\begin{aligned}
& \nabla_{y}\left(\lambda^{T} K\right)(x, y) \leqslant 0, \\
& \lambda>0, \lambda^{T} e=1 .
\end{aligned}
$$

\section{Dual Problem.}

(WD): Maximise $K(u, v)-\left[u^{T} \nabla_{x}\left(\lambda^{T} K\right)(u, v)\right] e$ Subject to

$$
\begin{array}{r}
\nabla_{x}\left(\lambda^{T} K\right)(u, v) \geqslant 0, \\
\lambda>0, \lambda^{T} e=1 .
\end{array}
$$

These are the symmetric dual problems formulated by Weir and Mond [12], with the omission of constraints $x \geqslant 0$ from (WP) and $v \geqslant 0$ from (WD). In the following analysis, we shall use $Z$ and $W$ for the set of feasible solutions of (WP) and (WD) respectively. If the variable $\lambda$ in the above problems (WP) and (WD) is fixed to be $\lambda_{0}$, we shall denote these problems by (WP $)_{\lambda 0}$ and (WD) $\lambda_{\lambda 0}$. Similar notations will be followd for Mond-Weir type symmetric dual problems discussed in Section 4.

ThEOREM 1. (Weak Duality.) Let $K(., y)$ be invex in $x$ with respect to $\eta$ and $-K(x,$.$) be invex in y$ with respect to $\xi$, with $\eta(x, u)+u \geqslant 0$ and $\xi(v, y)+y \geqslant 0$, whenever $(x, y, \lambda) \in Z$ and $(u, v, \lambda) \in W$. Then

$$
\lambda^{T}\left[K(x, y)-\left(y^{T} \nabla_{y}\left(\lambda^{T} K\right)(x, y)\right) e\right] \geqslant \lambda^{T}\left[K(u, v)-\left(u^{T} \nabla_{x}\left(\lambda^{T} K\right)(u, v)\right) e\right] .
$$

Proof: By invexity of $K(., y)$ and $-K(x,$.$) , we have$

$$
\lambda^{T} K(x, v)-\lambda^{T} K(u, v) \geqslant \eta^{T}(x, u) \nabla_{x}\left(\lambda^{T} K\right)(u, v),
$$


and

$$
-\lambda^{T} K(x, v)+\lambda^{T} K(x, y) \geqslant-\xi^{T}(v, y) \nabla_{y}\left(\lambda^{T} K\right)(x, y)
$$

Adding the above inequalities, we obtain

$$
\lambda^{T} K(x, y)-\lambda^{T} K(u, v) \geqslant \eta^{T}(x, u) \nabla_{x}\left(\lambda^{T} K\right)(u, v)-\xi^{T}(v, y) \nabla_{y}\left(\lambda^{T} K\right)(x, y) .
$$

From (3) and $\eta(x, u)+u \geqslant 0$, we have

implying

$$
(\eta(x, u)+u)^{T}\left(\nabla_{x}\left(\lambda^{T} K\right)(u, v)\right) \geqslant 0
$$

$$
\eta^{T}(x, u) \nabla_{x}\left(\lambda^{T} K\right)(u, v) \geqslant-u^{T} \nabla_{x}\left(\lambda^{T} K\right)(u, v)
$$

Also, from (1) and $\xi(v, y)+y \geqslant 0$, we have

$$
-\xi^{T}(v, y) \nabla_{y}\left(\lambda^{T} K\right)(x, y) \geqslant y^{T} \nabla_{y}\left(\lambda^{T} K\right)(x, y)
$$

From (7), (8), and (9), we obtain

$$
\lambda^{T} K(x, y)-\lambda^{T} K(u, v) \geqslant-u^{T} \nabla_{x}\left(\lambda^{T} K\right)(u, v)+y^{T} \nabla_{y}\left(\lambda^{T} K\right)(x, y),
$$

which gives

$$
\lambda^{T} K(x, y)-y^{T} \nabla_{y}\left(\lambda^{T} K\right)(x, y) \geqslant \lambda^{T} K(u, v)-u^{T} \nabla_{x}\left(\lambda^{T} K\right)(u, v) .
$$

Since $\lambda^{T} e=1$, the above inequality can be written as

$$
\lambda^{T} K(x, y)-\left(\lambda^{T} e\right) y^{T} \nabla_{y}\left(\lambda^{T} K\right)(x, y) \geqslant \lambda^{T} K(u, v)-\left(\lambda^{T} e\right) u^{T} \nabla_{x}\left(\lambda^{T} K\right)(u, v),
$$

that is,

$$
\lambda^{T}\left[K(x, y)-\left(y^{T} \nabla_{y}\left(\lambda^{T} K\right)(x, y)\right) e\right] \geqslant \lambda^{T}\left[K(u, v)-\left(u^{T} \nabla_{x}\left(\lambda^{T} K\right)(u, v)\right) e\right] .
$$

REMARK 1. If the invexity assumptions of Theorem 1 are replaced by convexity and concavity, the conditions $\eta(x, u)+u \geqslant 0$ and $\xi(v, y)+y \geqslant 0$ become $x \geqslant 0$ and $v \geqslant 0$. These conditions may be augmented to problems (WP) and (WD) respectively to obtain the pair of Wolfe type symmetric dual problems, considered by Mond and Weir [12].

The following theorem also serves to correct the proof of Theorem 2 in Weir and Mond [12] as while applying Fritz John conditions to (WP), the contraint $\lambda^{T} e=1$ has not been considered. 
Theorem 2. (Strong Duality.) Let the hypotheses of Theorem 1 be satisfied. Assume that

(A1): $\left(x_{0}, y_{0}, \lambda_{0}\right)$ is a weak efficient solution of (WP),

(A2): the Hessian matrix $\nabla_{y y}\left(\lambda_{0}^{T} K\right)\left(x_{0}, y_{0}\right)$ is positive or negative definite, and

(A3): the set $\left\{\nabla_{y} K^{1}\left(x_{0}, y_{0}\right), \nabla_{y} K^{2}\left(x_{0}, y_{0}\right), \ldots, \nabla_{y} K^{p}\left(x_{0}, y_{0}\right)\right\}$ is linearly independent.

Then $\left(x_{0}, y_{0}\right)$ is a properly efficient solution of $(W D)_{\lambda 0}$.

Proof: Since $\left(x_{0}, y_{0}, \lambda_{0}\right)$ is a weak efficient solution of (WP), by the Fritz John optimality conditions [3], there exist $\alpha \in R^{p}, \beta \in R^{m}, \gamma \in R^{p}$ and $\eta \in R$ such that

$$
\begin{gathered}
\nabla_{x}\left(\alpha^{T} K\right)+\nabla_{y x}\left(\lambda_{0}^{T} K\right)\left(\beta-\left(\alpha^{T} e\right) y_{0}\right)=0 \\
\left(\nabla_{y} K\right)\left(\alpha-\left(\alpha^{T} e\right) \lambda_{0}\right)+\nabla_{y y}\left(\lambda_{0}^{T} K\right)\left(\beta-\left(\alpha^{T} e\right) y_{0}\right)=0 \\
\left(\beta-\left(\alpha^{T} e\right) y_{0}\right)^{T} \nabla_{y} K^{i}-\gamma^{i}+\eta=0, i \in\{1,2, \ldots, p\} \\
\gamma^{T} \lambda_{0}=0 \\
\beta^{T} \nabla_{y}\left(\lambda_{0}^{T} K\right)=0 \\
(\alpha, \beta, \gamma) \geqslant 0, \\
(\alpha, \beta, \gamma, \eta) \neq 0
\end{gathered}
$$

with all derivatives evaluated at $\left(x_{0}, y_{0}\right)$. Since $\lambda_{0}>0$ and $\gamma \geqslant 0$, equation (13) yields $\gamma=0$. By multiplying (12) by $\left(\alpha^{i}-\left(\alpha^{T} e\right) \lambda_{0}^{i}\right), i \in\{1,2, \ldots, p\}$, summing the resulting expression for all $i$ and using $\lambda_{0}^{T} e=1$, we obtain

$$
\begin{aligned}
\left(\beta-\left(\alpha^{T} e\right) y_{0}\right)^{T} \sum_{i=1}^{p}\left(\nabla_{y} K^{i}\right)\left(\alpha^{i}-\left(\alpha^{T} e\right) \lambda_{0}^{i}\right) & =-\eta \sum_{i=1}^{p}\left(\alpha^{i}-\left(\alpha^{T} e\right) \lambda_{0}^{i}\right) \\
& =-\eta\left(\sum_{i=1}^{p} \alpha^{i}-\left(\alpha^{T} e\right) \sum_{i=1}^{p} \lambda_{0}^{i}\right)=0 .
\end{aligned}
$$

That is,

$$
\left(\beta-\left(\alpha^{T} e\right) y_{0}\right)^{T} \nabla_{y} K\left(x_{0}, y_{0}\right)\left(\alpha-\left(\alpha^{T} e\right) \lambda_{0}\right)=0
$$

On multiplying (11) by $\left(\beta-\left(\alpha^{T} e\right) y_{0}\right)^{T}$ and using (17), we have

$$
\left(\beta-\left(\alpha^{T} e\right) y_{0}\right)^{T} \nabla_{y y}\left(\lambda_{0}^{T} K\right)\left(\beta-\left(\alpha^{T} e\right) y_{0}\right)=0
$$


which, in view of assumption (A2), leads to the relation

$$
\beta-\left(\alpha^{T} e\right) y_{0}=0
$$

Therefore from (12) it follows that $\eta=0$.

If $\alpha=0$, then (19) yields $\beta=0$. Consequently $(\alpha, \beta, \gamma, \eta)=0$, contradicting the Fritz John condition (16). Hence, $\alpha \gtrsim 0$ and

$$
\alpha^{T} e>0
$$

From equations (11) and (19),

$$
\nabla_{y} K\left(x_{0}, y_{0}\right)\left(\alpha-\left(\alpha^{T} e\right) \lambda_{0}\right)=0
$$

which by assumption (A3) gives

$$
\alpha=\left(\alpha^{T} e\right) \lambda_{0}
$$

Now using relations (10), (19), (20) and (21), we get

$$
\nabla_{x}\left(\lambda_{0}^{T} K\right)\left(x_{0}, y_{0}\right)=\left(\nabla_{x}\left(\alpha^{T} K\right)\left(x_{0}, y_{0}\right)\right) /\left(\alpha^{T} e\right)=0
$$

Hence $\left(x_{0}, y_{0}\right)$ is feasible for (WD) $)_{\lambda 0}$. Also from (22)

$$
x_{0}^{T} \nabla_{x}\left(\lambda_{0}^{T} K\right)\left(x_{0}, y_{0}\right)=0
$$

and from (14), (19) and (20),

$$
y_{0}^{T} \nabla_{y}\left(\lambda_{0}^{T} K\right)\left(x_{0}, y_{0}\right)=0
$$

Therefore

$$
x_{0}^{T} \nabla_{x}\left(\lambda_{0}^{T} K\right)\left(x_{0}, y_{0}\right)=y_{0}^{T} \nabla_{y}\left(\lambda_{0}^{T} K\right)\left(x_{0}, y_{0}\right)=0
$$

that is, the two objectives are equal.

Now we show proper efficiency of $\left(x_{0}, y_{0}\right)$ for (WD) $)_{\lambda 0}$ by exhibiting a contradition. If $\left(x_{0}, y_{0}\right)$ is not efficient, then there exists $\left(u_{0}, v_{0}\right)$ feasible for (WD) ${ }_{\lambda 0}$, that is, $\left(u_{0}, v_{0}, \lambda_{0}\right) \in W$ such that

$$
K\left(u_{0}, v_{0}\right)-\left(u_{0}^{T} \nabla_{x}\left(\lambda_{0}^{T} K\right)\left(u_{0}, v_{0}\right)\right) e \gtrsim K\left(x_{0}, y_{0}\right)-\left(x_{0}^{T} \nabla_{x}\left(\lambda_{0}^{T} K\right)\left(x_{0}, y_{0}\right)\right) e
$$


In view of $(23)$, it follows that

$$
K\left(u_{0}, v_{0}\right)-\left(u_{0}^{T} \nabla_{x}\left(\lambda_{0}^{T} K\right)\left(u_{0}, v_{0}\right)\right) e \gtrsim K\left(x_{0}, y_{0}\right)-\left(y_{0}^{T} \nabla_{y}\left(\lambda_{0}^{T} K\right)\left(x_{0}, y_{0}\right)\right) e
$$

Hence

$$
\lambda_{0}^{T}\left[K\left(u_{0}, v_{0}\right)-\left(u_{0}^{T} \nabla_{x}\left(\lambda_{0}^{T} K\right)\left(u_{0}, v_{0}\right)\right) e\right]>\lambda_{0}^{T}\left[K\left(x_{0}, y_{0}\right)-\left(y_{0}^{T} \nabla_{y}\left(\lambda_{0}^{T} K\right)\left(x_{0}, y_{0}\right)\right) e\right]
$$

which contadicts Theorem 1. If $\left(x_{0}, y_{0}\right)$ were an improperly efficient solution of (WD) $\lambda_{\lambda 0}$, then for some feasible $\left(u_{0}, v_{0}\right)$ and some $i$,

$$
\left[K^{i}\left(u_{0}, v_{0}\right)-\left(u_{0}^{T} \nabla_{x}\left(\lambda_{0}^{T} K\right)\left(u_{0}, v_{0}\right)\right)\right]>\left[K^{i}\left(x_{0}, y_{0}\right)-\left(x_{0}^{T} \nabla_{x}\left(\lambda_{0}^{T} K\right)\left(x_{0}, y_{0}\right)\right)\right]
$$

and

$$
\begin{gathered}
{\left[\left\{K^{i}\left(u_{0}, v_{0}\right)-\left(u_{0}^{T} \nabla_{x}\left(\lambda_{0}^{T} K\right)\left(u_{0}, v_{0}\right)\right)\right\}-\left\{K^{i}\left(x_{0}, y_{0}\right)-\left(x_{0}^{T} \nabla_{x}\left(\lambda_{0}^{T} K\right)\left(x_{0}, y_{0}\right)\right)\right\}\right]} \\
>M\left[\left\{K^{j}\left(x_{0}, y_{0}\right)-\left(x_{0}^{T} \nabla_{x}\left(\lambda_{0}^{T} K\right)\left(x_{0}, y_{0}\right)\right)\right\}\right. \\
\left.-\left\{K^{j}\left(u_{0}, v_{0}\right)-\left(u_{0}^{T} \nabla_{x}\left(\lambda_{0}^{T} K\right)\left(u_{0}, v_{0}\right)\right)\right\}\right]
\end{gathered}
$$

for any $M>0$, and all $j$ satisfying

$$
\left[K^{j}\left(x_{0}, y_{0}\right)-\left(x_{0}^{T} \nabla_{x}\left(\lambda_{0}^{T} K\right)\left(x_{0}, y_{0}\right)\right)\right]>\left[K^{j}\left(u_{0}, v_{0}\right)-\left(u_{0}^{T} \nabla_{x}\left(\lambda_{0}^{T} K\right)\left(u_{0}, v_{0}\right)\right)\right]
$$

This means that

$$
\left[\left\{K^{i}\left(u_{0}, v_{0}\right)-\left(u_{0}^{T} \nabla_{x}\left(\lambda_{0}^{T} K\right)\left(u_{0}, v_{0}\right)\right)\right\}-\left\{K^{i}\left(x_{0}, y_{0}\right)-\left(x_{0}^{T} \nabla_{x}\left(\lambda_{0}^{T} K\right)\left(x_{0}, y_{0}\right)\right)\right\}\right]
$$

can be made arbitrary large whereas

$$
\left[\left\{K^{j}\left(x_{0}, y_{0}\right)-\left(x_{0}^{T} \nabla_{x}\left(\lambda_{0}^{T} K\right)\left(x_{0}, y_{0}\right)\right)\right\}-\left\{K^{j}\left(u_{0}, v_{0}\right)-\left(u_{0}^{T} \nabla_{x}\left(\lambda_{0}^{T} K\right)\left(u_{0}, v_{0}\right)\right)\right\}\right]
$$

is finite for all $j \neq i$. Since $\lambda_{0}=\left(\lambda_{0}^{1}, \lambda_{0}^{2}, \ldots, \lambda_{0}^{p}\right)>0$, we get

$$
\begin{aligned}
\lambda_{0}^{i}\left[\left\{K^{i}\left(u_{0}, v_{0}\right)-\right.\right. & \left.\left.\left(u_{0}^{T} \nabla_{x}\left(\lambda_{0}^{T} K\right)\left(u_{0}, v_{0}\right)\right)\right\}-\left\{K^{i}\left(x_{0}, y_{0}\right)-\left(x_{0}^{T} \nabla_{x}\left(\lambda_{0}^{T} K\right)\left(x_{0}, y_{0}\right)\right)\right\}\right] \\
>\sum_{j \neq i} \lambda_{0}^{j}\left[\left\{K^{j}\left(x_{0}, y_{0}\right)-\right.\right. & \left.\left(x_{0}^{T} \nabla_{x}\left(\lambda_{0}^{T} K\right)\left(x_{0}, y_{0}\right)\right)\right\} \\
& \left.-\left\{K^{j}\left(u_{0}, v_{0}\right)-\left(u_{0}^{T} \nabla_{x}\left(\lambda_{0}^{T} K\right)\left(u_{0}, v_{0}\right)\right)\right\}\right]
\end{aligned}
$$

or

$\lambda_{0}^{T}\left[K\left(u_{0}, v_{0}\right)-\left(u_{0}^{T} \nabla_{x}\left(\lambda_{0}^{T} K\right)\left(u_{0}, v_{0}\right)\right) e\right]>\lambda_{0}^{T}\left[K\left(x_{0}, y_{0}\right)-\left(y_{0}^{T} \nabla_{y}\left(\lambda_{0}^{T} K\right)\left(x_{0}, y_{0}\right)\right) e\right]$

(using (23) and $\lambda_{0}^{T} e=1$ ). This again contradicts Theorem 1 .

A converse duality theorem may be merely stated as its proof would run analogously to that of Theorem 2 . 
TheOREM 3. (Converse Duality.) Let the hypotheses of Theorem 1 be satisfied. Assume that

(B1): $\left(u_{0}, v_{0}, \lambda_{0}\right)$ is a weak efficient solution of (WD),

(B2): the Hessian matrix $\nabla_{x x}\left(\lambda_{0}^{T} K\right)\left(u_{0}, v_{0}\right)$ is positive or negative definite, and

(B3): the set $\left\{\nabla_{x} K^{1}\left(u_{0}, v_{0}\right), \nabla_{x} K^{2}\left(u_{0}, v_{0}\right), \ldots, \nabla_{x} K^{p}\left(u_{0}, v_{0}\right)\right\}$ is linearly independent.

Then $\left(u_{0}, v_{0}\right)$ is a properly efficient solution of $(W P)_{\lambda 0}$.

\section{MOND-WEIR TYPE SYMMETRIC DUALITY}

In this section, we consider the following pair of Mond-Weir type symmetric dual problems:

Primal Problem.

(MP): Minimise $K(x, y)$

Subject to

$$
\begin{aligned}
\nabla_{y}\left(\lambda^{T} K\right)(x, y) & \leqslant 0, \\
y^{T} \nabla_{y}\left(\lambda^{T} K\right)(x, y) & \geqslant 0, \\
\lambda & >0
\end{aligned}
$$

DuAl Problem.

(MD): Maximise $K(u, v)$

Subject to

$$
\begin{aligned}
\nabla_{x}\left(\lambda^{T} K\right)(u, v) & \geqslant 0, \\
u^{T} \nabla_{x}\left(\lambda^{T} K\right)(u, v) & \leqslant 0, \\
\lambda & >0 .
\end{aligned}
$$

When $x \geqslant 0$ is adjoined to (MP) and $v \geqslant 0$ to (MD), the above problems represent the pair of symmetric dual problems considered by Weir and Mond [12].

In the following analysis, we shall use $H$ and $G$ for the set of feasible solutions of the problems (MP) and (MD) respectively.

ThEOREM 4. (Weak Duality.) Let $(x, y, \lambda) \in H$ and $(u, v, \lambda) \in G$. Let $\lambda^{T} K(., y)$ be pseudo-invex with respect to $\eta$ for fixed $y$, and $-\lambda^{T} K(x,$.$) be pseudo-invex with$ respect to $\xi$ for fixed $x$, with $\eta(x, u)+u \geqslant 0$ and $\xi(v, y)+y \geqslant 0$. Then

$$
\lambda^{T} K(x, y) \geqslant \lambda^{T} K(u, v)
$$


Proof: From (27) and $\eta(x, u)+u \geqslant 0$, we have

$$
\eta^{T}(x, u)\left(\nabla_{x}\left(\lambda^{T} K\right)(u, v)\right) \geqslant-u^{T} \nabla_{x}\left(\lambda^{T} K\right)(u, v) \geqslant 0,
$$

(using (28)) which, in view of the pseudo-invexity of $\lambda^{T} K(., y)$, gives

$$
\lambda^{T} K(x, v) \geqslant \lambda^{T} K(u, v) .
$$

From (24) and $\xi(v, y)+y \geqslant 0$, it follows that

$$
\xi^{T}(v, y) \nabla_{y}\left(\lambda^{T} K\right)(x, y) \leqslant-y^{T} \nabla_{y}\left(\lambda^{T} K\right)(x, y) \leqslant 0,
$$

(using (25)). This, because of the pseudo-invexity of $-\lambda^{T} K(x,$.$) implies$

$$
\lambda^{T} K(x, v) \leqslant \lambda^{T} K(x, y) .
$$

The relations (30) and (31) yield

$$
\lambda^{T} K(x, y) \geqslant \lambda^{T} K(u, v) .
$$

Theorem 5. (Strong Duality.) Assume that the hypotheses of Theorem 4 are satisfied. Let

(C1): $\left(x_{0}, y_{0}, \lambda_{0}\right)$ be a weak efficient solution for $(M P)$,

(C2): $\nabla_{y y}\left(\lambda_{0}^{T} K\right)\left(x_{0}, y_{0}\right)$ be positive or negative definite, and

$(\mathrm{C} 3)$ : the set $\left\{\nabla_{y} K^{1}\left(x_{0}, y_{0}\right), \ldots, \nabla_{y} K^{p}\left(x_{0}, y_{0}\right)\right\}$ be linearly independent.

Then $\left(x_{0}, y_{0}\right)$ is a properly efficient solution of $(M D)_{\lambda 0}$

Proof: Since $\left(x_{0}, y_{0}, \lambda_{0}\right)$ is a weak efficient solution of (MP), by the Fritz John optimality theorem of [3], there exist $\alpha \in R^{p}, \beta \in R^{m}, \eta \in R$ and $\gamma \in R^{p}$ such that

$$
\begin{aligned}
\nabla_{x}\left(\alpha^{T} K\right)+\nabla_{y x}\left(\lambda_{0}^{T} K\right)\left(\beta-\eta y_{0}\right) & =0, \\
\left(\nabla_{y} K\right)\left(\alpha-\eta \lambda_{0}\right)+\nabla_{y y}\left(\lambda_{0}^{T} K\right)\left(\beta-\eta y_{0}\right) & =0, \\
\left(\beta-\eta y_{0}\right)^{T}\left(\nabla_{y} K\right)-\gamma & =0, \\
\beta^{T} \nabla_{y}\left(\lambda_{0}^{T} K\right) & =0, \\
\eta y_{0}^{T} \nabla_{y}\left(\lambda_{0}^{T} K\right) & =0, \\
\gamma^{T} \lambda_{0} & =0, \\
(\alpha, \beta, \gamma, \eta) & \geqslant 0, \\
(\alpha, \beta, \gamma, \eta) & \neq 0,
\end{aligned}
$$


with all derivatives evaluated at $\left(x_{0}, y_{0}\right)$. In view of $\lambda_{0}>0$ and $\gamma \geqslant 0$, it readily follows from (37) that $\gamma=0$. Therefore from (34), we have

$$
\left(\beta-\eta y_{0}\right)^{T}\left(\nabla_{y} K\right)=0
$$

Multiplying (33) by $\left(\beta-\eta y_{0}\right)^{T}$,

$$
\left(\beta-\eta y_{0}\right)^{T}\left(\nabla_{y} K\right)\left(\alpha-\eta \lambda_{0}\right)+\left(\beta-\eta y_{0}\right)^{T} \nabla_{y y}\left(\lambda_{0}^{T} K\right)\left(\beta-\eta y_{0}\right)=0
$$

which, in conjunction with (40), implies

$$
\left(\beta-\eta y_{0}\right)^{T} \nabla_{y y}\left(\lambda_{0}^{T} K\right)\left(\beta-\eta y_{0}\right)=0 .
$$

Since $\nabla_{y y}\left(\lambda_{0}^{T} K\right)$ is positive or negative definite, we have

$$
\beta-\eta y_{0}=0
$$

Equation (33) together with (41) and the linear independence of $\left\{\nabla_{y} K^{1}\left(x_{0}, y_{0}\right), \ldots\right.$, $\left.\nabla_{y} K^{p}\left(x_{0}, y_{0}\right)\right\}$, yields

$$
\alpha=\eta \lambda_{0}
$$

Suppose $\eta=0$, then from (42), $\alpha=0$. Also, from (41) we have $\beta=0$. Therefore $(\alpha, \beta, \gamma, \eta)=0$, contradicting (39). Hence $\eta>0$. Also, from (32), (41) and (42) along with $\eta>0$, we obtain

which implies

$$
\begin{aligned}
\nabla_{x}\left(\lambda_{0}^{T} K\right)\left(x_{0}, y_{0}\right) & =0, \\
x_{0}^{T} \nabla_{x}\left(\lambda_{0}^{T} K\right)\left(x_{0}, y_{0}\right) & =0 .
\end{aligned}
$$

Hence $\left(x_{0}, y_{0}\right)$ is feasible for $(\mathrm{MD})_{\lambda 0}$, and the two objectives become equal there. Now, similar to the proof of Theorem 2 it can be shown that $\left(x_{0}, y_{0}\right)$ is a property efficient solution for $(\mathrm{MD})_{\lambda 0}$.

As in the preceding section, here too, a converse duality theorem may be stated without proof.

Theorem 6. (Converse Duality.) Assume that the hypotheses of Theorem 4 are satisfied. Let

(D1): $\left(u_{0}, v_{0}, \lambda_{0}\right)$ be a weak efficient solution for $(M D)$,

(D2): $\quad \nabla_{x x}\left(\lambda^{T} K\right)\left(u_{0}, v_{0}\right)$ be positive or negative definite, and

(D3): the set $\left\{\nabla_{x} K^{1}\left(u_{0}, v_{0}\right), \ldots, \nabla_{x} K^{p}\left(u_{0}, v_{0}\right)\right\}$ be linearly independent.

Then $\left(u_{0}, v_{0}\right)$ is a properly efficient solution of $(M P)_{\lambda 0}$. 


\section{Self Duality}

A mathematical problem is said to be self dual if it is formally identical with its dual, that is, if the dual is recast in the form of the primal, the new problem so obtained is the same as the primal. In general (WP) and (WD) are not self dual without an added restriction on $K$. The vector function $K: R^{n} \times R^{n} \rightarrow R$ is said to be skew symmetric if for all $x, y \in R^{n}$,

$$
K(y, x)=-K(x, y), \text { that is, } K^{i}(y, x)=-K^{i}(x, y), i \in\{1,2, \ldots, p\} .
$$

Now recasting the dual problem (WD) as a minimisation problem:

$$
\begin{aligned}
& \left(\mathrm{WD}_{0}\right): \quad \text { Minimise }-K(u, v)+\left[u^{T} \nabla_{x}\left(\lambda^{T} K\right)(u, v)\right] e \\
& \text { Subject to } \\
& \nabla_{x}\left(\lambda^{T} K\right)(u, v) \geqslant 0 \text {, } \\
& \lambda>0, \lambda^{T} e=1 .
\end{aligned}
$$

Since $\nabla_{x} K(u, v)=-\nabla_{y} K(v, u)$, the problem $\left(\mathrm{WD}_{0}\right)$ reduces to,

Minimise $K(v, u)-\left[u^{T} \nabla_{y}\left(\lambda^{T} K\right)(v, u)\right] e$

Subject to

$$
\begin{array}{r}
\nabla_{y}\left(\lambda^{T} K\right)(v, u) \leqslant 0, \\
\lambda>0, \lambda^{T} e=1 .
\end{array}
$$

This shows that $\left(\mathrm{WD}_{0}\right)$ is formally identical to (WP), that is, the objective and constraint functions are identical. Hence (WP) is self dual. Consequently, the feasibility of $(x, y, \lambda)$ for (WP) implies the feasibility of $(y, x, \lambda)$ for (WD) and conversely.

We now state the following self duality theorems. Their proofs are similar to the corresponding results in [12].

THEOREM 7. (Self Duality.) Let $K$ be skew symmetric. Then (WP) is self dual. If also (WP) and (WD) are dual problems and $\left(x_{0}, y_{0}, \lambda_{0}\right)$ is a joint properly efficient solution, then so is $\left(y_{0}, x_{0}, \lambda_{0}\right)$ and the common optimal value of the objective functions is 0 .

THEOREM 8. (Self Duality.) Let $K$ be skew symmetric. Then $(M P)$ is self dual. If also (MP) and (MD) are dual problems and $\left(x_{0}, y_{0}, \lambda_{0}\right)$ is a joint properly efficient solution, then so is $\left(y_{0}, x_{0}, \lambda_{0}\right)$ and

$$
\begin{gathered}
K\left(x_{0}, y_{0}\right)=K\left(y_{0}, x_{0}\right)=0 . \\
\text { REFERENCES }
\end{gathered}
$$

[1] J.M. Borwein, Optimization with respect to partial ordering, D.Phil. Thesis (Oxford University, 1974). 
[2] S. Chandra and I. Husain, 'Symmetric dual nondifferentiable programs', Bull. Austral. Math. Soc. 24 (1981), 295-307.

[3] B.D. Craven, 'Lagrangian conditions and quasiduality', Bull. Austral. Math. Soc. 16 (1977), 325-339.

[4] G.B. Dantzig, E. Eisenberg and R.W. Cottle, 'Symmetric dual nonlinear programs', Pacific J. Math 15 (1965), 809-812.

[5] W.S. Dorn, 'A symmetric dual theorem for quadratic programs', J. Oper. Res. Soc. Japan 2 (1960), 93-97.

6] A.M. Geoffrion, 'Proper efficiency and the theory of vector maximization', J. Math. Anal. Appl. 22 (1968), 618 630.

[7] M.A. Hanson, 'On sufficiency of the Kuhn-Tucker conditions', J. Math. Anal. Appl. 80 (1981), 545-550.

[8] B. Mond and R.W. Cottle, 'Self duality in mathematical programming', SIAM J. Appl. Math. 14 (1966), 420-423.

[9] B. Mond and M.A. Hanson, 'Symmetric duality for variational problems', J. Math. Anal. Appl. 23 (1968), 161-172.

[10] B. Mond and T. Weir, 'Generalized concavity and duality', in Generalized concavity and duality in optimization and economics, (S. Schaible and W.T. Ziemba, Editors) (Academic Press, New York, 1981), pp. 263-279.

[11] I. Smart and B. Mond, 'Symmetric duality with invexity in variational problems', J. Math. Anal. Appl. 152 (1992), 536-545.

[12] T. Weir and B. Mond, 'Symmetric and self duality in multiple objective programming', Asia-Pacific J. Oper. Res. 5 (1988), 124-133.

School of Basic \& Applied Sciences

Thapar Institute of Engineering \& Technology

Patiala - 147001

India

Department of Mathematics \& Statistics

University of Kashmir

Hazratbal, Srinagar-190 006

India
Department of Mathematics Regional Engineering College Hazratbal, Srinagar - 190006 India 